\title{
Serum osteocalcin levels are inversely associated with plasma glucose and body mass index in healthy Chinese women
}

\author{
Wei-wei HU ${ }^{1, \#}$, Yao-hua KE ${ }^{1, \#}$, Jin-wei HE, Wen-zhen FU, Yu-juan LIU, Di CHEN², Zhen-lin ZHANG* \\ ${ }^{1}$ Metabolic Bone Disease and Genetic Research Unit, the Department of Osteoporosis and Bone Disease, Shanghai Key Clinical Center \\ for Metabolic Disease, Shanghai Jiao Tong University Affiliated Sixth People's Hospital, Shanghai 200233, China; ${ }^{2}$ Department of \\ Biochemistry, Rush University Medical Center, Chicago, IL 60612, USA
}

Aim: Osteocalcin, a biochemical marker of bone formation, has been suggested to be involved in the regulation of energy metabolism. The aim of this study was to investigate the possible association between serum osteocalcin and markers of glucose and lipid metabolism in a large sample of healthy Chinese women.

Methods: A total of 2032 healthy Chinese women in Shanghai, aged 20-94 (including 1396 discovery-study subjects and 636 postmenopausal women for a reduplication analysis) were recruited. Their serum osteocalcin, calcium and the relevant measurements were analyzed. A Spearman correlation analysis was performed between osteocalcin and the other markers of energy metabolism including triglyceride, total cholesterol, fasting plasma glucose (FPG), serum insulin, body mass index and homeostasis model assessment-insulin resistance. Separate multiple regression analyses were performed with data from the discovery and reduplication subjects to determine whether serum osteocalcin concentration was an independent predictor of the glucose or lipid metabolism markers.

Results: For the discovery-study subjects, serum osteocalcin was found to be negatively associated with weight ( $r=-0.08, P=0.002)$, $\operatorname{BMI}(r=-0.13, P<0.001)$ and FPG $(r=-0.13, P=0.001)$. Similar results were also found in the reduplication subjects (weight: $r=-0.19$, $P=0.016$; BMI: $r=-0.23, P=0.003$; FPG: $r=-0.28, P<0.001)$. In the multiple regression analysis, serum osteocalcin was revealed as a potential independent predictor for FPG $(\beta=-0.07$ and -0.210 for discovery and reduplication, respectively, $P<0.01)$ and $B M I(\beta=-0.127$ and -0.299 for discovery and reduplication, respectively, $P<0.01$ ).

Conclusion: Serum osteocalcin is negatively associated with weight BMI and FPG in healthy Chinese women. Therefore, osteocalcin might contribute to obesity and diabetes.

Keywords: osteocalcin; glucose metabolism; BMl; insulin resistance; Chinese women

Acta Pharmacologica Sinica (2014) 35: 1521-1526; doi: 10.1038/aps.2014.92; published online Oct 202014

\section{Introduction}

Osteocalcin, which is produced and secreted specifically by osteoblasts, is one of the most abundant noncollagen proteins in bone matrix ${ }^{[1]}$. Its measurement has been used as a biochemical marker of bone formation and bone turnover ${ }^{[2]}$. However, numerous studies have also evaluated the potential role of osteocalcin as a predictor and surrogate marker of fracture risk or osteoporosis ${ }^{[3,4]}$. Recent studies have suggested that osteocalcin may also be involved in the regulation of

\footnotetext{
\# The first two authors contributed equally to this work.

* To whom correspondence should be addressed.

E-mail ZZL2002@medmail.com.cn

Received 2014-05-02 Accepted 2014-08-20
}

energy metabolism ${ }^{[5,6]}$. Lee et $\mathrm{l}^{[6]}$ demonstrated that mice lacking osteocalcin exhibited hyperglycemia, obesity, decreased $\beta$-cell proliferation, glucose intolerance and insulin resistance. Ex vivo studies have found that osteocalcin stimulates the expression and action of CyclinD1, insulin, and adiponectin. Another animal study ${ }^{[5]}$, which focused on the mechanism of the novel function of osteocalcin, revealed that mice lacking FoxO1 in osteoblasts had increased pancreatic $\beta$-cell proliferation, insulin secretion and insulin sensitivity. FoxO1, which is a member of the Forkhead family of transcription factors, was indicated as a key transcriptional modulator of the endocrine function of the skeleton in regulating glucose metabolism. These findings in mice provided evidence of a novel role of the skeleton in glucose and lipid homeostasis, which is regulated 
by osteocalcin.

A number of studies have evaluated the complex interactions between the skeleton and glucose and energy homeostasis in humans ${ }^{[7-15]}$. Osteocalcin was found to be inversely associated with fasting plasma glucose $(\mathrm{FPG})^{[7,9,10]}, \mathrm{HbA} 1 \mathrm{c}^{[7,12]}$, body mass index $(\mathrm{BMI})^{[10,13]}$, fat mass ${ }^{[10]}$, measures of insulin resistance $^{[8,14]}$, and measures of lipid metabolism ${ }^{[12,15]}$. In these same studies, osteocalcin was positively associated with adiponectin $^{[11]}$ and measures of insulin sensitivity ${ }^{[7,15]}$. Among these studies, the positive relationship between osteocalcin and markers of metabolic phenotype were revealed mostly in the elderly population or subjects with diabetes mellitus. Recently, a study found that serum osteocalcin levels were inversely correlated with visceral fat area in Chinese men ${ }^{[16]}$. However, the results of the studies on the association between osteocalcin and lipid metabolism are inconsistent ${ }^{[7,13,15]}$. Therefore, it is necessary to perform more human osteocalcin-metabolism association studies with larger sample sizes and healthy populations to provide insights into the relation between serum osteocalcin levels and glucose and lipid metabolism.

In this study, we performed a cross-sectional analysis to investigate the possible association between osteocalcin and markers of glucose and lipid metabolism in a large sample of healthy Chinese females.

\section{Materials and methods Subjects}

The study was approved by the Ethics Committee of the Shanghai Jiao Tong University Affiliated Sixth People's Hospital. Between February and March 2009, we recruited 2243 women (aged 20-96 years) living in Shanghai from several community centers. But only 1396 people can independently complete the questionnaire and physical examination. Of these 1396 women, 39 failed the initial screening, leaving 1357 subjects with detected serum osteocalcin. All the subjects belonged to the Chinese Han ethnic group and signed informed consent documents before entering the study ${ }^{[17]}$. All subjects completed a questionnaire, providing information on age, medical history, etc. A physical checkup and routine serum examination were performed. All of the subjects were found to be in good health. None of the subjects suffered from diseases known to affect bone metabolism or used drugs affecting bone metabolism. Subjects who had a history of diabetes mellitus or fasting plasma glucose (FPG) $>7.0 \mathrm{mmol} / \mathrm{L}$ were not eligible for the study.

For the reduplication analysis, 636 healthy ambulatory postmenopausal women (aged 55-75 years) were recruited for a national clinical trial on the prevention of bone fracture in a population with a high risk of osteoporosis. The exclusion criteria were the same as in our previous study, including the presence of the following: (1) serious residual effects from cerebral vascular disease; (2) diabetes mellitus; (3) hyperthyroidism; (4) chronic renal disease or renal lithiasis; (5) serious chronic liver disease; (6) significant chronic gastrointestinal disease; (7) rheumatoid arthritis or collagen disease; (8) cancer;
(9) corticosteroid or anticoagulation therapy at pharmacological levels; (10) evidence of other metabolic or inherited bone diseases; (11) treatment with calcium $>600 \mathrm{mg} / \mathrm{d}$ or $\mathrm{VitD}>600$ $\mathrm{IU} / \mathrm{d}$; (12) therapy with bisphosphonate or fluoride in the past 1 year; or (13) therapy with calcitonin, estrogen or SERMs in the past 3 years $^{[18]}$.

\section{Blood measurement}

The blood samples were collected after an 8-h or greater overnight fast for measurement of serum calcium (Ca), phosphate $(\mathrm{P})$, alkaline phosphatase (ALP), triglyceride (TG), total cholesterol (TC), and fasting plasma glucose (FPG). The electrochemiluminescence immunoassay (ECLIA, Elecsys autoanalyzer, Roche Diagnostics GmbH, Germany) was used to measure the serum insulin (intra and inter assay coefficients of variation of $1.5 \%-2.0 \%$ and $2.1 \%-2.8 \%$, respectively). Serum 25-hydroxyvitamin D [25(OH)D], parathyroid hormone (PTH), $\beta$-crosslinked C-telopeptide of type 1 collagen (CTX), osteocalcin in form of N-MID, and N-terminal procollagen of type 1 collagen (P1NP) were measured using an automated Roche electro-chemiluminescence system (Roche Diagnostic Gmbh, Germany). The intra- and inter-assay coefficients of variation (CVs) for $25(\mathrm{OH}) \mathrm{D}$ were $5.6 \%$ and $8.0 \%$, respectively. The lower limit of detection for $25(\mathrm{OH}) \mathrm{D}$ was $4 \mathrm{ng} / \mathrm{mL}(10 \mathrm{mmol} / \mathrm{L})$. The intraand interassay $\mathrm{CVs}$ were $1.4 \%$ and $2.9 \%$ for $\mathrm{PTH}, 2.5 \%$ and $3.5 \%$ for $\beta$-CTX, $2.9 \%$ and $4.0 \%$ for osteocalcin, and $2.3 \%$ and $2.8 \%$ for P1NP, respectively ${ }^{[17,19,20]}$.

\section{Other measurements}

A physical examination including the measurement of height and weight was performed for each participant. Body mass index (BMI) was calculated as weight divided by squared height $\left(\mathrm{kg} / \mathrm{m}^{2}\right)$. The assessment of insulin sensitivity was estimated by homeostasis model assessment-insulin resistance (HOMA-IR) based on FPG and insulin measurements as follows: HOMA-IR=[fasting serum insulin $(\mathrm{mU} / \mathrm{L}) \times \mathrm{FPG}$ $(\mathrm{mmol} / \mathrm{L})] / 22.5^{[14]}$

\section{Statistical analysis}

All statistical analyses were performed using SPSS version 19 software (SPSS Inc of IBM, USA). Skewed variables were transformed to natural logarithms for the formal analyses. The ANOVA test was used to compare the general characteristics between the quartiles of serum osteocalcin. Spearman correlation analysis was performed between osteocalcin and the other factors; the Spearman correlation coefficients for the glucose, lipid and bone metabolism parameters were calculated to determine which factors would be included in the final model (data not shown). In the final model, multivariate regression analysis was performed to determine the associations between serum osteocalcin as the independent variable and FPG, insulin, HOMA-IR, TG, TC, and BMI after adjusting for age, BMI, $\mathrm{Ca}, \mathrm{P}, \mathrm{ALP}, \mathrm{TG}, \mathrm{TC}$, and PTH (No adjustment was made for $\mathrm{BMI}, \mathrm{TG}$, or TC when they were included as outcome variables in the model).

In the 636 postmenopausal female subjects included as 
reduplicated samples, the spearman correlation and multivariate regression analysis were repeated to compare against the results from the discovery study. For all statistical analyses, $P<0.05$ was considered significant.

\section{Results}

\section{The clinical characteristics of the study subjects}

Among the 1396 female participants of the discovery study, 39 women who met the exclusion criteria were removed from the analysis. The general characteristics of the subjects in the discovery study and the reduplication group were summarized in Table 1. For the reduplication analysis, the mean age, BMI, and FPG of 636 healthy postmenopausal women were $62.8 \pm 5.8$ year, $24.7 \pm 3.3 \mathrm{~kg} / \mathrm{m}^{2}$, and $4.74 \pm 0.59 \mathrm{mmol} / \mathrm{L}$, respectively.

The discovery study subjects were divided into quartiles by their serum osteocalcin concentration (Table 2). BMI varied inversely with the osteocalcin quartiles $(P<0.01)$. The FPG level in the lowest osteocalcin quartiles was significantly higher than in the other quartiles in both the pair-wise and

Table 1. Anthropometric characteristics and other variables in the discovery group and reduplication group.

\begin{tabular}{lll}
\hline Characteristics & Discovery group & Reduplication group \\
\hline$n$ & 1357 & 636 \\
Age $($ year $)$ & $38.5 \pm 12.8$ & $62.8 \pm 5.8$ \\
$\mathrm{BMI}$ & $22.4 \pm 3.1$ & $24.7 \pm 3.3$ \\
$\mathrm{Ca}(\mathrm{mmol} / \mathrm{L})$ & $2.27 \pm 0.14$ & $2.23 \pm 0.16$ \\
$\mathrm{P}(\mathrm{mmol} / \mathrm{L})$ & $1.09 \pm 0.16$ & $1.02 \pm 0.11$ \\
$\mathrm{ALP}(\mathrm{U} / \mathrm{L})$ & $59.3 \pm 16.5$ & $72.3 \pm 12.9$ \\
$\mathrm{PTH}(\mathrm{pg} / \mathrm{mL})$ & $33.7(26.3-43.4)$ & $52.8(21.6-65.9)$ \\
$\mathrm{TG}(\mathrm{mmol} / \mathrm{L})$ & $0.84(0.60-1.28)$ & \\
TC $(\mathrm{mmol} / \mathrm{L})$ & $4.51 \pm 0.89$ & \\
FPG $(\mathrm{mmol} / \mathrm{L})$ & $4.66 \pm 0.92$ & $4.74 \pm 0.59$ \\
Insulin $(\mu \mathrm{IU} / \mathrm{mL})$ & $6.76(4.83-9.49)$ & $6.85(4.07-10.53)$ \\
$\mathrm{HOMA}-\mathrm{IR}$ & $1.45(0.96-2.01)$ & $1.58(0.90-2.27)$ \\
\hline
\end{tabular}

overall comparisons. No significant differences or tendencies in the serum insulin, HOMA-IR, TG, or TC were found according to the quartiles of osteocalcin.

\section{The correlation between serum osteocalcin and other para- meters}

We studied the association between serum osteocalcin and other body or serum/plasma parameters in both the discovery study and the reduplication subjects (Table 3). For the discovery study subjects, serum osteocalcin was negatively associated with weight, BMI and FPG $(r=-0.08, r=-0.13$, and $r=-0.13$, respectively, $P<0.01)$; the negative associations with weight, BMI and FPG were also found in the reduplication subjects $(r=-0.19, r=-0.23$, and $r=-0.28$, respectively, $P<0.05)$. The correlation was significantly stronger between serum osteocalcin and FPG in the postmenopausal women than in the overall group containing both young and elderly subjects $(r=-0.28$ and $r=-0.13, P<0.01)$. However, no significant association between serum osteocalcin and insulin/HOMA-IR was detected in either the discovery or the reduplication groups. Furthermore, no association was found between the two markers of lipid

Table 3. Correlation analyses of serum osteocalcin concentration vs other parameters in both subjects.

\begin{tabular}{lcccc}
\hline & \multicolumn{2}{c}{ Discovery study } & \multicolumn{2}{c}{ Reduplication study } \\
Variable & $r$ & $P$ & $r$ & $P$ \\
\hline Age & -0.01 & 0.83 & 0.03 & 0.63 \\
Height & 0.04 & 0.16 & 0.04 & 0.63 \\
Weight & -0.08 & 0.002 & -0.19 & 0.016 \\
BMI & -0.13 & $<0.001$ & -0.23 & 0.003 \\
TG & -0.03 & 0.22 & - & - \\
TC & -0.04 & 0.08 & - & - \\
FPG & -0.13 & 0.001 & -0.28 & $<0.001$ \\
Insulin & -0.004 & 0.87 & 0.08 & 0.326 \\
HOMA-IR & -0.019 & 0.40 & 0.03 & 0.72 \\
\hline
\end{tabular}

Table 2. Differences in the values of general characteristics in serum osteocalcin concentration quartiles. ${ }^{\mathrm{c}} P<0.01 \mathrm{vs} 16.8-21.6 \mathrm{ng} / \mathrm{mL}$ group. ${ }^{\mathrm{f}} P<0.01$ vs $>21.6 \mathrm{ng} / \mathrm{mL}$ group. ${ }^{\mathrm{h}} \mathrm{P}<0.05$ vs $13.2-16.8 \mathrm{ng} / \mathrm{mL}$ group.

\begin{tabular}{|c|c|c|c|c|c|c|}
\hline Characteristics & Overall & $<13.2$ & \multicolumn{2}{|c|}{ Osteocalcin quartiles $(\mathrm{ng} / \mathrm{mL})$} & $>21.6$ & $P$ value \\
\hline$n$ & 1357 & 355 & 335 & 327 & 340 & \\
\hline $\mathrm{BMI}$ & $22.4 \pm 3.1$ & $23.8 \pm 3.5^{\mathrm{ch}}$ & $22.9 \pm 3.1^{c}$ & $22.1 \pm 3.0^{f}$ & $21.2 \pm 3.0$ & $<0.05$ \\
\hline $\mathrm{Ca}(\mathrm{mmol} / \mathrm{L})$ & $2.27 \pm 0.14$ & $2.26 \pm 0.14$ & $2.27 \pm 0.13$ & $2.27 \pm 0.13$ & $2.29 \pm 0.14$ & 0.127 \\
\hline $\mathrm{P}(\mathrm{mmol} / \mathrm{L})$ & $1.09 \pm 0.16$ & $1.09 \pm 0.16$ & $1.08 \pm 0.15$ & $1.10 \pm 0.17$ & $1.10 \pm 0.16$ & 0.210 \\
\hline $\mathrm{TG}(\mathrm{mmol} / \mathrm{L})$ & $0.84(0.60-1.28)$ & $0.79(0.58-1.15)$ & $0.86(0.61-1.34)$ & $0.82(0.60-1.29)$ & $0.84(0.59-1.25)$ & 0.197 \\
\hline $\mathrm{TC}(\mathrm{mmol} / \mathrm{L})$ & $4.51 \pm 0.89$ & $4.52 \pm 0.90$ & $4.55 \pm 0.82$ & $4.49 \pm 0.91$ & $4.51 \pm 0.87$ & 0.213 \\
\hline $\mathrm{FPG}(\mathrm{mmol} / \mathrm{L})$ & $4.66 \pm 0.92$ & $4.92 \pm 0.56^{c h}$ & $4.74 \pm 0.61^{\circ}$ & $4.63 \pm 0.55^{f}$ & $4.51 \pm 0.56$ & $<0.05$ \\
\hline Insulin $(\mu \mathrm{IU} / \mathrm{mL})$ & $6.76(4.83-9.49)$ & $6.84(4.82-9.71)$ & $6.95(4.83-9.75)$ & $6.57(4.83-9.16)$ & $6.61(4.76-9.36)$ & 0.691 \\
\hline HOMA-IR & $1.45(0.96-2.01)$ & $1.51(0.95-1.97)$ & $1.47(0.98-2.00)$ & 1.39 (0.96-1.95) & $1.42(0.98-2.17)$ & 0.113 \\
\hline
\end{tabular}

Notes: Normally distributed data are shown as meanıstandard. Data that are not normally distributed are shown as medians and inter-quartile ranges (in parentheses). 
metabolism, TG and TC, and serum osteocalcin in the discovery study subjects.

\section{The multiple regression analyses for osteocalcin as the inde- pendent predictor}

To determine whether the serum osteocalcin concentration was an independent predictor of the markers of glucose or lipid metabolism, we performed separate multiple regression analyses in the discovery and reduplication subjects (Table 4). In the discovery subjects, we found a negative association between serum osteocalcin and FPG after adjusting for age, BMI, Ca, P, ALP, PTH, TG, and TC $(\beta=-0.07, P<0.01)$. Also a negative association between serum osteocalcin and $\mathrm{BMI}$ was found after adjusting for age, FPG, Ca, P, ALP, PTH, TG, and TC $(\beta=-0.127, P<0.01)$. Serum osteocalcin levels were also inversely associated with FPG and BMI $(\beta=-0.210$ and $\beta=-0.299, P<0.01)$ in the reduplication study. However, no evidence was found that serum osteocalcin played a role as an independent predictor of serum insulin, HOMA-IR, TG, or TC in either the discovery or the reduplication subjects.

Table 4. Serum osteocalcin concentration as a potential independent predictor for the parameters of energy metabolism. ${ }^{\circ} P<0.01$.

\begin{tabular}{llc}
\hline Dependent variable & $\begin{array}{c}\beta \text {-value for } \\
\text { Discovery study }\end{array}$ & $\begin{array}{c}\beta \text {-value for } \\
\text { Reduplication study }\end{array}$ \\
\hline FPG $(95 \% \mathrm{Cl})$ & $-0.07(-0.047 ;-0.001)^{\mathrm{c}}$ & $-0.210(-0.129 ;-0.018)^{\mathrm{c}}$ \\
Insulin $(95 \% \mathrm{Cl})$ & $-0.03(-0.162 ; 0.057)$ & $0.115(-0.092 ; 0.554)$ \\
HOMA-IR $(95 \% \mathrm{Cl})$ & $-0.047(-0.191 ; 0.034)$ & $-0.076(-0.177 ; 0.495)$ \\
BMI $(95 \% \mathrm{Cl})$ & $-0.127(-0.068 ;-0.023)^{\mathrm{c}}$ & $-0.299(-9.654 ;-3.134)^{\mathrm{c}}$ \\
TG $(95 \% \mathrm{Cl})$ & $0.028(-0.053 ; 0.139)$ & - \\
TC $(95 \% \mathrm{Cl})$ & $0.001(-0.034 ; 0.035)$ & - \\
\hline
\end{tabular}

Standardized $\beta$-values for osteocalcin from linear regression analyses.

\section{Discussion}

Osteocalcin, which contains 49 amino acids (including 3 $\mathrm{\gamma}$-carboxylated glutamic acid residues), is produced and excreted by osteoblasts ${ }^{[21,22]}$. As a classic biochemical marker for bone turnover, the circulating osteocalcin has been measured widely in the diagnosis and treatment of metabolic bone diseases ${ }^{[23]}$. However, recent studies have suggested that osteocalcin may also be involved in the regulation of energy metabolism. Lee et $a l^{[6]}$ showed that mice lacking the osteotesticular protein tyrosine phosphatase (OST-PTP) were hypoglycemic and were protected from obesity and glucose intolerance. OST-PTP, which is encoded by the enterococcal surface protein gene $(E s p)$, has been found to downregulate the activity of osteocalcin ${ }^{[24]}$.

In the present study, we revealed that serum osteocalcin was negatively associated with BMI in Chinese healthy women. The mechanisms associating fat mass with osteocalcin are not fully understood. It has been suggested that osteocalcin concentrations are modulated by the adipocytokine leptin. In humans, lower circulating osteocalcin levels were found in overweight $^{[25]}$ and diabetic subjects ${ }^{[26]}$ compared with normal weight individuals.

In our study, we also found a negative association, which was also confirmed in the reduplication analysis, between serum osteocalcin and BMI, which was a phenotype of obesity. In a previous study, Bao et $a l^{[16]}$ also found that visceral fat area and percent body fat were independent factors negatively associated with serum osteocalcin levels in Chinese men. In animal studies, after 6 weeks of a high-fat diet, the body weight of the $\mathrm{Esp}^{-/-}$mice increased at a slower rate than did the wild type (WT) mouse ${ }^{[6]}$. In another mouse model, the $\mathrm{OCn}^{-/}$mouse, in which the Osteocalcin gene was knocked out, was obese and had significantly increased fat mass and numbers of adipocytes. The results of our study are in agreement with others who have shown the inverse association between osteocalcin and BMI. However, in addition to BMI, fat mass and lean mass, which are measured by dual energy $x$-ray absorptiometry (DXA), are also useful markers reflecting body composition and obesity. It is unfortunate that we were not able to test fat mass and lean mass by DXA to further confirm the association between serum osteocalcin and the phenotypes of obesity in this population. However, a recent study found that visceral fat area was inversely associated with serum osteocalcin levels in Chinese men, which is in agreement with our results ${ }^{[16]}$.

A significantly lower osteocalcin concentration has been found in patients with diabetes mellitus than in healthy individuals ${ }^{[27-29]}$; this phenomenon may be the result of a decrease in the number of osteoblasts due to hyperglycemia ${ }^{[30,31]}$. However, according to Lee et al's study ${ }^{[6]}$, osteocalcin may also regulate glucose metabolism. Previous studies in humans have attempted to reveal the relationship between osteocalcin and glucose metabolism ${ }^{[7-10,12,14,15]}$. In the present study, we revealed that serum osteocalcin was negatively associated with FPG. However, we found no further evidence to support the theory that total serum osteocalcin plays a role as an independent predictor of the phenotypes of insulin resistance in either the discovery or reduplication subjects. Our results are similar to that of a study of elderly Swedish men ${ }^{[10]}$, in which Kindblom et al found that plasma osteocalcin was inversely related to the plasma glucose, not insulin resistance.

Based on the limited number of existing studies, the relationship between osteocalcin and lipid metabolism in humans remains unclear. In the present study, we analyzed the independent impact of osteocalcin on two phenotypes of lipid metabolism, TG and TC, in the discovery subjects. No association was found to prove that osteocalcin affected these two markers of lipid metabolism. In the previous human studies, Im et $a l^{[13]}$ failed to find an association between serum osteocalcin and blood lipids in postmenopausal women, and Zhou et $a l^{[7]}$ revealed that serum osteocalcin was positively associated with high-density lipoprotein cholesterol in Chinese elderly men and TG in premenopausal women. The current study conducted in a large sample of the healthy population provides useful data on the relationship between osteocalcin and lipid metabolism in humans. 
The results of the present study suggest that circulating osteocalcin is involved in the regulation of obesity and blood glucose, but not in the regulation of insulin resistance and lipid metabolism. Previous studies have found increased insulin sensitivity in the $E s p^{-/}$mouse suggesting that circulating osteocalcin enhances insulin sensitivity through the increased expression of adiponectin ${ }^{[6,32]}$. Adiponectin is a polypeptide which modulates glucose regulation and fatty acid catabolism ${ }^{[33]}$. It has been reported that adiponectin concentration is inversely correlated with body fat percentage in adults ${ }^{[34]}$. Recently, adiponectin was also shown to have a positive association with serum osteocalcin in postmenopausal women with diabetes mellitus. We investigated the relationship between osteocalcin and insulin resistance by calculating the subject's HOMA-IR, which was found to be inversely associated with serum osteocalcin in the previous studies ${ }^{[9]}$. Our negative results may reflect the imprecision of HOMA-IR as a surrogate measure of insulin resistance. The detection of the adiponectin concentration would have helped further elucidate the relationship between osteocalcin and insulin resistance in this study.

The further study of the mechanism of osteocalcin's regulation of insulin resistance may contribute to the treatment of a series of energy metabolism diseases. A recent animal study ${ }^{[5]}$ showed that mice lacking Foxo1 only in osteoblasts had increased pancreatic $\beta$-cell proliferation, insulin secretion and insulin sensitivity. Foxo1 was indicated to be an upstream regulator that controls glucose metabolism through the stimulation of osteocalcin and the inhibition of Esp expression in osteoblasts.

On the other hand, all of the animal studies provide evidence that only uncarboxylated osteocalcin influences energy metabolism in mice ${ }^{[6,32]}$. Nevertheless, osteocalcin is only able to bind calcium in its carboxylated form, which depends on vitamin $\mathrm{K}$ and carbon dioxide. In women, (elevated/ decreased) uncarboxylated osteocalcin levels were found to be a risk factor for bone fracture ${ }^{[35]}$ and were associated with a (increase or decrease) in vitamin $\mathrm{K}$ levels. This research suggests that uncarboxylated osteocalcin plays an adverse role in bone metabolism and energy metabolism. Further study of the maximally-beneficial level of circulating osteocalcin should be performed in the future.

The present study has several limitations. Our measurement of osteocalcin was N-mid osteocalcin, which cannot be separated into its carboxylated and uncarboxylated forms. Additional markers of the phenotypes of energy metabolism such as HbA1c, fat mass, and lean mass were not investigated in this study. The present study used a cross-sectional design with healthy subjects; it is necessary for us to perform a longterm prospective analysis to confirm the study results.

However, the present study still revealed valuable information about the association between osteocalcin and energy metabolism. In a large sample of the healthy Chinese population, we found that the serum osteocalcin concentration was inversely associated with plasma glucose and was a strong predictor of BMI; these results were also confirmed in post- menopausal Chinese women as a reduplication analysis. Our data supported the finding that osteocalcin is involved in the regulation of energy metabolism. Therefore, osteocalcin might be a promising link between obesity and diabetes.

\section{Acknowledgements}

This study was supported by the National Natural Science Foundation of China (NSFC) (Grant Noxs 81370978, 81070692 and 81170803), Academic Leaders in Health Sciences in Shanghai (XBR2011014, 2014CB942903, SHDC12013115, CSTC2013jcyjC00009), National Basic Research Program of China (Grant No 2014CB942903 to Zhen-lin ZHANG) and Program for Outstanding Medical Academic Leader (051).

\section{Author contribution}

Wei-wei HU designed the study, carried out all statistical analyses and drafted the manuscript. Zhen-lin ZHANG conceived and designed the study and revised the manuscript. Di CHEN revised the manuscript. Jin-wei HE guided the work of the genetics laboratory and guaranteed and confirmed the quality of the genetic data. Wen-zhen FU guided the work of the genetics laboratory. Yao-hua KE carried out the fieldwork including sample and data collection. Yu-juan LIU coordinated the DNA sample collection. All authors read and approved the final manuscript.

\section{References}

1 Price PA. Gla-containing proteins of bone. Connect Tissue Res 1989; 21: 51-7; discussion 57-60.

2 Delmas PD. Biochemical markers of bone turnover for the clinical investigation of osteoporosis. Osteoporos Int 1993; 3 Suppl 1: 81-6.

3 Luukinen H, Kakonen SM, Pettersson K, Koski K, Laippala P, Lovgren T, et al. Strong prediction of fractures among older adults by the ratio of carboxylated to total serum osteocalcin. J Bone Miner Res 2000; 15: 2473-8.

4 Lenora J, Ivaska KK, Obrant KJ, Gerdhem P. Prediction of bone loss using biochemical markers of bone turnover. Osteoporos Int 2007; 18: 1297-305.

5 Rached MT, Kode A, Silva BC, Jung DY, Gray S, Ong H, et al. FoxO1 expression in osteoblasts regulates glucose homeostasis through regulation of osteocalcin in mice. J Clin Invest 2010; 120: 357-68.

6 Lee NK, Sowa H, Hinoi E, Ferron M, Ahn JD, Confavreux C, et al. Endocrine regulation of energy metabolism by the skeleton. Cell 2007; 130: 456-69.

7 Zhou M, Ma X, Li H, Pan X, Tang J, Gao Y, et al. Serum osteocalcin concentrations in relation to glucose and lipid metabolism in Chinese individuals. Eur J Endocrinol 2009; 161: 723-9.

8 Shea MK, Gundberg CM, Meigs JB, Dallal GE, Saltzman E, Yoshida M, et al. Gamma-carboxylation of osteocalcin and insulin resistance in older men and women. Am J Clin Nutr 2009; 90: 1230-5.

9 Pittas AG, Harris SS, Eliades M, Stark P, Dawson-Hughes B. Association between serum osteocalcin and markers of metabolic phenotype. J Clin Endocrinol Metab 2009; 94: 827-32.

10 Kindblom JM, Ohlsson C, Ljunggren O, Karlsson MK, Tivesten A, Smith $\mathrm{U}$, et al. Plasma osteocalcin is inversely related to fat mass and plasma glucose in elderly Swedish men. J Bone Miner Res 2009; 24: 785-91.

11 Kanazawa I, Yamaguchi T, Yamamoto M, Yamauchi M, Kurioka S, 
Yano S, et al. Serum osteocalcin level is associated with glucose metabolism and atherosclerosis parameters in type 2 diabetes mellitus. J Clin Endocrinol Metab 2009; 94: 45-9.

12 Kanazawa I, Yamaguchi T, Sugimoto T. Relationship between bone biochemical markers versus glucose/lipid metabolism and atherosclerosis; a longitudinal study in type 2 diabetes mellitus. Diabetes Res Clin Pract 2011; 92: 393-9.

13 Im JA, Yu BP, Jeon JY, Kim SH. Relationship between osteocalcin and glucose metabolism in postmenopausal women. Clin Chim Acta 2008; 396: 66-9.

14 Iki M, Tamaki J, Fujita Y, Kouda K, Yura A, Kadowaki E, et al. Serum undercarboxylated osteocalcin levels are inversely associated with glycemic status and insulin resistance in an elderly Japanese male population: Fujiwara-kyo Osteoporosis Risk in Men (FORMEN) Study. Osteoporos Int 2012; 23: 761-70.

15 Fernandez-Real JM, Izquierdo M, Ortega F, Gorostiaga E, GomezAmbrosi J, Moreno-Navarrete JM, et al. The relationship of serum osteocalcin concentration to insulin secretion, sensitivity, and disposal with hypocaloric diet and resistance training. J Clin Endocrinol Metab 2009; 94: 237-45.

16 Bao Y, Ma X, Yang R, Wang F, Hao Y, Dou J, et al. Inverse relationship between serum osteocalcin levels and visceral fat area in Chinese men. J Clin Endocrinol Metab 98: 345-51.

17 Tao MF, Zhang Z, Ke YH, He JW, Fu WZ, Zhang CQ, et al. Association of serum 25-hydroxyvitamin $D$ with insulin resistance and beta-cell function in a healthy Chinese female population. Acta Pharmacol Sin 2013; 34: 1070-4.

18 Yue H, He JW, Ke YH, Zhang H, Wang C, Hu WW, et al. Association of single nucleotide polymorphism Rs2236518 in PRDM16 gene with BMI in Chinese males. Acta Pharmacol Sin 2013; 34: 710-6.

19 Hu WW, Zhang Z, He JW, Fu WZ, Wang C, Zhang H, et al. Establishing reference intervals for bone turnover markers in the healthy shanghai population and the relationship with bone mineral density in postmenopausal women. Int J Endocrinol 2013; 2013: 513925.

20 Zhang Z, He JW, Fu WZ, Zhang CQ, Zhang ZL. An analysis of the association between the vitamin $D$ pathway and serum 25-hydroxyvitamin D levels in a healthy Chinese population. J Bone Miner Res 2013; 28: 1784-92.

21 Hauschka PV, Lian JB, Cole DE, Gundberg CM. Osteocalcin and matrix Gla protein: vitamin K-dependent proteins in bone. Physiol Rev 1989; 69: 990-1047.

22 Lian J, Stewart C, Puchacz E, Mackowiak S, Shalhoub V, Collart D, et al. Structure of the rat osteocalcin gene and regulation of vitamin D-dependent expression. Proc Natl Acad Sci U S A 1989; 86: 1143-7. 23 Yoshimura N, Muraki S, Oka H, Kawaguchi H, Nakamura K, Akune T.
Biochemical markers of bone turnover as predictors of osteoporosis and osteoporotic fractures in men and women: 10-year follow-up of the Taiji cohort. Mod Rheumatol 2011; 21: 608-20.

24 Mauro $\amalg$, Olmsted EA, Skrobacz BM, Mourey RJ, Davis AR, Dixon JE. Identification of a hormonally regulated protein tyrosine phosphatase associated with bone and testicular differentiation. J Biol Chem 1994; 269: 30659-67.

25 Holecki M, Zahorska-Markiewicz B, Janowska J, Nieszporek T, Wojaczynska-Stanek K, Zak-Golab A, et al. The influence of weight loss on serum osteoprotegerin concentration in obese perimenopausal women. Obesity (Silver Spring) 2007; 15: 1925-9.

26 Bouillon R, Bex M, Van Herck E, Laureys J, Dooms L, Lesaffre E, et al. Influence of age, sex, and insulin on osteoblast function: osteoblast dysfunction in diabetes mellitus. J Clin Endocrinol Metab 1995; 80: 1194-202.

27 Verhaeghe J, Suiker AM, Visser WJ, Van Herck E, Van Bree R, Bouillon R. The effects of systemic insulin, insulin-like growth factor-I and growth hormone on bone growth and turnover in spontaneously diabetic BB rats. J Endocrinol 1992; 134: 485-92.

28 Pietschmann P, Schernthaner G, Woloszczuk W. Serum osteocalcin levels in diabetes mellitus: analysis of the type of diabetes and microvascular complications. Diabetologia 1988; 31: 892-5.

29 Krakauer JC, McKenna MJ, Buderer NF, Rao DS, Whitehouse FW, Parfitt AM. Bone loss and bone turnover in diabetes. Diabetes 1995; 44: 775-82.

30 Gerdhem P, Isaksson A, Akesson K, Obrant KJ. Increased bone density and decreased bone turnover, but no evident alteration of fracture susceptibility in elderly women with diabetes mellitus. Osteoporos Int 2005; 16: 1506-12.

31 Rico H, Hernandez ER, Cabranes JA, Gomez-Castresana F. Suggestion of a deficient osteoblastic function in diabetes mellitus: the possible cause of osteopenia in diabetics. Calcif Tissue Int 1989; 45: 71-3.

32 Ferron M, Hinoi E, Karsenty G, Ducy P. Osteocalcin differentially regulates beta cell and adipocyte gene expression and affects the development of metabolic diseases in wild-type mice. Proc Natl Acad Sci U S A 2008; 105: 5266-70.

33 Diez JJ, Iglesias P. The role of the novel adipocyte-derived hormone adiponectin in human disease. Eur J Endocrinol 2003; 148: 293300.

34 Ukkola O, Santaniemi M. Adiponectin: a link between excess adiposity and associated comorbidities? J Mol Med (Berl) 2002; 80: 696-702.

35 Vergnaud P, Garnero P, Meunier PJ, Breart G, Kamihagi K, Delmas $P D$. Undercarboxylated osteocalcin measured with a specific immunoassay predicts hip fracture in elderly women: the EPIDOS Study. J Clin Endocrinol Metab 1997; 82: 719-24. 